\title{
Releasing Dye Encapsulated in Proteinaceous Microspheres on Conductive Fabrics by Electric Current
}

\author{
Ulyana Shimanovich, ${ }^{\dagger}$ Ilana Perelshtein, ${ }^{\dagger}$ Artur Cavaco-Paulo, ${ }^{\ddagger}$ and Aharon Gedanken* ${ }^{\dagger}$ \\ ${ }^{\dagger}$ Department of Chemistry and Kanbar Laboratory for Nanomaterials Bar-Ilan University Center for Advanced Materials and \\ Nanotechnology, Bar-Ilan University, Ramat-Gan 52900 (Israel). \\ ${ }^{\ddagger}$ Textile Engineering Department, University of Minho, P-4800058 Guimaraes, Portugal
}

\begin{abstract}
The current paper reports on the relase properties of conductive fabrics coated with proteinaceous microspheres containing a dye. The release of the dye was achieved by passing an electric current through the fabric. The conductivity of the polyester fibers resulted from nanosilver (Ag NPs) coated on the surface of these fibers. Both types of coatings (nanosilver coating and the coating of the proteinaceous microspheres) were performed using high-intensity ultrasonic waves. Two different types of dyes, hydrophilic RBBR (Remazol Brilliant Blue R) and hydrophobic ORO (Oil Red $\mathrm{O}$ ), were encapsulated inside the microspheres (attached

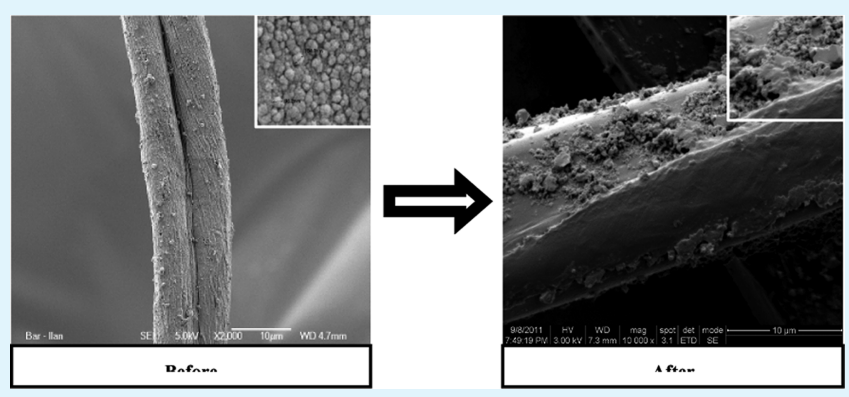
to the surface of polyester) and then released by applying an electric current. The Proteinaceous Microsphere (PM)-coated conductive fabrics could be used in medicine for drug release. The encapsulated dye can be replaced with a drug that could be released from the surface of fabrics by applying a low voltage.
\end{abstract}

KEYWORDS: sonochemistry, microsphere, nanosilver, surface coating, dye release, electrical current

\section{INTRODUCTION}

Sonochemistry is the application of ultrasound to chemical reactions and processes. The mechanism causing sonochemical effects in liquids is the phenomenon of acoustic cavitation. Sonochemical irradiation has been proven as an effective method for the synthesis of nanophased materials, ${ }^{1}$ as well as for the deposition and insertion of nanoparticles on/into mesoporous ceramic and polymer supports. ${ }^{2-4}$ Our previous work $^{5}$ described coating nylon, polyester, and cotton fabrics with nanosilver to impart antimicrobial properties to the textiles, using high intensity ultrasound. Silver provides excellent conductivity that yields both electromagnetic shielding (EMI shielding) and radio frequency shielding (RFI) to the coated surface. Electrically conductive silver coated yarn and fibers are used as antistatic elements in carpets; carpet fabrics; primary and secondary carpet backings; furnishing fabrics; and in industrial applications, such as filter fabrics, needle felts, webbing conveyor belt fabrics, and balloon nets. They also find use in work wear, sterile garments, in the electronic industry, operating theater clothing, and antiexplosion wear (refineries, the chemical industry, and in the manufacture of explosives). In general, small quantities of coated silver make highly conductive fibers and yarns, and are also sufficient for many antistatic applications.

In addition, we have used sonochemical radiation as a technique for attaching drug-loaded proteinaceous microspheres to cotton and polyester fabrics. ${ }^{6,7}$ The formation, characterization and properties of sonochemically made, proteinaceous microspheres (PMs) were recently reviewed. ${ }^{8}$
According to the mechanism proposed for the sonochemical formation of PM, the spheres are formed by chemically crosslinking the cysteine residues of the protein with the $\mathrm{HO}_{2}$ radical formed around a micrometer-sized gas bubble or a nonaqueous droplet. The chemical cross-linking is responsible for the formation of the spheres, and is a direct result of the chemical effects of ultrasound radiation on an aqueous medium. The creation of the microbubbles is a short process that lasts 3 $\min ^{9-11}$

In the current research, we tried to combine two types of coatings (nanosilver coating and coating the silver-coated fabrics with an upper layer of proteinaceous microspheres) in order to produce fabrics with "release control" functions. The idea was to first coat fabrics with silver nanoparticles (NPs) in order to create conductive textiles and then to attach PMs loaded with a "drugs" to the "conductive" fabrics. The release of the drug from the inner part of the bonded PMs was achieved by an applying electric current. For the initial studies we encapsulated two types of dyes in the PMs instead of a "drug": (1) RBBR (Remaszol Brilliant Blue R), ${ }^{12}$ an hydrophilic dye, and (2) ORO (Oil Red O $)^{13}$ hydrophobic dye. The dyes were chosen to be encapsulated due to their different nature. By studying the behavior of these two dyes during sonochemical encapsulation process we can predict the behavior of potential drugs which will suggested for encapsulation (judging from

\footnotetext{
Received: February 12, 2012

Accepted: $\quad$ May 3, 2012

Published: May 3, 2012
} 


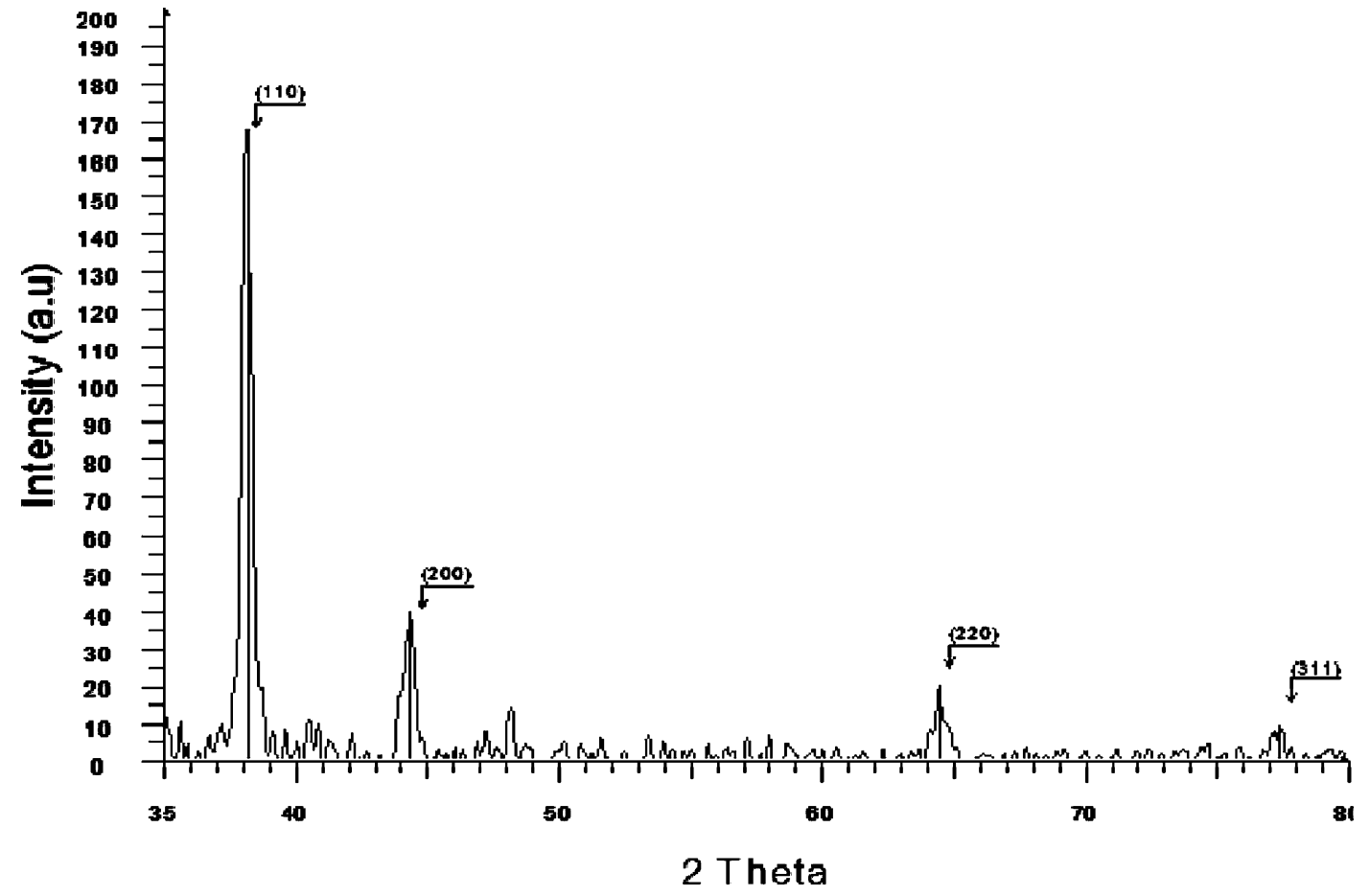

Figure 1. XRD pattern of Ag nanoparticles deposited sonochemically on a polyester fabric. Reprinted with permission from ref 15 . Copyright 2008 Nanotechnology Publisher.

their hydrophilicity or hydrophobicity). Two types of polyester fabrics were coated with silver nanoparticles, mesh-polyester and regular polyester. The creation and the anchoring of the dye-loaded microbubbles to the "conductive" fabrics were performed by a one-step sonochemical reaction. The coated polyester was analyzed and characterized by light and electronic microscopy, dynamic light scattering, and UV spectroscopy. The conductivity of silver-coated fabrics was tested using the Van der Pauw technique.

\section{EXPERIMENTAL SECTION}

2.1. Sonochemical Coating of Silver Nanoparticles on Polyester Textiles. All the chemical reagents, of chemical grade were purchased from Aldrich and used without further purification. In a typical reaction, a $0.03 \mathrm{M}$ solution of water and ethylene glycol (EG) $(10: 1 \mathrm{v} / \mathrm{v})$ of $\mathrm{AgNO} 3$ was purged under $\mathrm{Ar}$ for $1 \mathrm{~h}$ in order to remove traces of $\mathrm{O}_{2}$ /air. The purging was done in the presence of the fabric (meshed and regular pieces of polyester $5 \times 5 \mathrm{~cm}$ ). The difference between the regular and the meshed polyester fabrics is in density of the fibers. In the next stage, the solution was irradiated for $1 \mathrm{~h}$ with a high intensity ultrasonic horn ( $\mathrm{Ti}$ horn, $20 \mathrm{kHz}, 600 \mathrm{~W}$ at $70 \%$ efficiency) under a flow of Ar. A 25\% aqueous solution of ammonia (molar ratio $\mathrm{NH}_{3}: \mathrm{AgNO}_{3}=2: 1$ ) was added to the reaction slurry during the first $10 \mathrm{~min}$ of sonication. The sonication flask was placed in a cooling bath keeping a constant temperature of $30^{\circ} \mathrm{C}$ during the sonication. At the end of the reaction, the color of the fabric changed from white to gray. The product was first washed thoroughly with water to remove traces of ammonia, then with ethanol, and dried under vacuum.

2.2. Attaching Microsphere-Containing Dyes to Conductive Fabrics Using Ultrasound Radiation. A BSA protein (albumin, bovine fraction v, Sigma) was used for the creation of PMs and the attachment of the BSA spheres to conductive (silver coated) polyester fabrics. In order to attach BSA microspheres containing dye to polyester fabrics, dodecane $(6.7 \mathrm{~mL}, 98.0 \%$ Fluka) was layered over 10 $\mathrm{mL}$ of a $5 \% \mathrm{w} / \mathrm{v}$ aqueous BSA solution with a piece $(5 \times 5 \mathrm{~cm}$. $)$ of $\mathrm{Ag}$ coated polyester fabric. Two types of dyes were used in those reactions: RBBR (Remazol Brilliant BlueR - hydrophilic dye, Sigma
Aldrich) and ORO (Oil Red O - hydrophobic dye, Sigma Aldrich). An $0.01 \%$ concentration of a dye solution was prepared before the sonochemical reaction. In the case of RBBR dye, the dye was first dissolved in water solution; in the case of ORO, the dye was first dissolved in dodecane.

$0.01 \%$ of the dye solution was added to the above-mentioned precursor mixture. The solution was sonicated for $3 \mathrm{~min}$ with a highintensity ultrasonic probe (Sonic and Materials, VC-600, $20 \mathrm{kHz}, 0.5$ in a $\mathrm{Ti}$ horn, at $30 \%$ amplitude). The bottom of the high-intensity ultrasonic horn was positioned at the aqueous-organic interface, employing an acoustic power of $\sim 58 \mathrm{~W} / \mathrm{cm}^{2}$ with an initial temperature of $22{ }^{\circ} \mathrm{C}$ in the reaction cell. An ice-cooling bath was used to keep the temperature constant. At the end of the reaction, the bandage was washed 3 times with $5 \mathrm{~mL}$ of water to remove the residue of the unbound microspheres and the pristine protein molecules.

2.3. Characterization Methods. The silver content in the fabrics was determined by volumetric titration with $\mathrm{KSCN}$ according to the Folgard method ${ }^{14}$ after dissolving the sonicated product in $\mathrm{HNO}_{3}$. The X-ray diffraction (XRD) pattern of the product was measured with a Bruker D8 diffractometer (Karlsruhe, Germany) with $\mathrm{Cu} \mathrm{K} \alpha$ radiation. The particle morphology and the size of the noble metal nanoparticles were studied with a high-resolution scanning electron microscope, HR-SEM (JEOL-JSN 7000F).

The size and electrical charge of PMs were measured by dynamic scattering technique. Dynamic light scattering (DLS) measurements and z-potential measurements were carried out on an ALV/CGS-3 compact goniometer system equipped with an ALV/LSE-5003 light scattering electronic and multiple s digital correlator, and a $632.8 \mathrm{~nm}$ JDSU laser 1145P. DLS and z-potential experiments were carried out on a doubly diluted as-separated PM solution, i.e., the PMs were removed after the sonication, and were diluted with an equal amount of doubly distilled water (DDW). Each measurement took $10 \mathrm{~s}$; particle distribution and electrical charge distribution were obtained by averaging over ten DLS measurements.

The distribution of the total amount of dye (encapsulated, not encapsulated and the residue in the solution) was determined by absorption measurements, as explained below. The UV-visible studies of the amount of dyes were based on measurements conducted using a UV-vis spectrophotometer (absorption wavelengths of RBBR 
absorption $=592 \mathrm{~nm}$; ORO absorption $=640 \mathrm{~nm}$ ). The calculations were based on the Beer-Lambert law: $A=\varepsilon c l$. A molar extinction coefficient of $\operatorname{RBBR}\left(\varepsilon_{592}=6170 \mathrm{~cm}^{-1} \mathrm{M}^{-1}\right)$ was used to compute the amount of encapsulated RBBR dye. The molar extinction coefficient of ORO is $\left(\varepsilon_{640}=4000 \mathrm{~cm}^{-1} \mathrm{M}^{-1}\right)$. The calculations were performed as follows. The absorption of individual dye molecules (not encapsulated in the microspheres) removed from the fabrics' surface was measured. By adding the amount of the "free" dye (RBBR/ORO that was not encapsulated in microspheres, but is attached to the silver-coated bandage), the amount of encapsulated dye, and the amount remaining in the aqueous solution after the sonication, we should get a total amount of the RBBR/ORO used in the coating process.

The conductivity of silver-coated fabrics was tested using the Van der Pauw technique. The resistivity test was performed on a Keithly model 6517 electrometer/high resistance meter.

\section{RESULTS AND DISCUSSION}

The first stage of the current investigation was to impart conductive properties to the textiles. This was achieved by the sonochemical deposition of silver NPs on the polyester fabric. The main challenge in achieving conductivity is to obtain a homogeneous, high density, nanoparticle coating We have found that by modifying reaction conditions such as: reaction time, initial concentration of the precursor, and temperature, we can control the particle size, concentration of the coating on the fabric surface, and the homogeneity. The fabric with the best conductive properties was used for further experimentation, as described below (Part 2.1.), has a concentration of $\sim 15$ wt $\%$ silver.

The crystallinity of the sonochemically deposited silver NPs on polyester was probed by XRD measurementS (Figure 1). The pattern indicates that the silver is crystalline, and the diffraction peaks match a face-centered cubic (fcc) phase of $\mathrm{Ag}$ (PDF: 4-783). The peaks at $2 \theta=38.03,44.23,64.39$, and $77.32^{\circ}$ are assigned to the (111), (200), (220), and (311) reflection lines of fcc $\mathrm{Ag}$ particles, respectively. No peaks characteristic of any impurities were detected.

Two types of polyester fabrics were coated with silver nanoparticles, mesh polyester and regular polyester. The basic method used to determine the current of a conductive sample is a two-step process; first, a test voltage is applied to the sample and the subsequent resistivity is measured. Once the voltage and the resistivity are measured, the current is calculated by the $\mathrm{Ohm}$ equation. The range of voltage values that was applied for resistivity measurements for regular silver-coated polyester fabric is from $-1.3 \mathrm{~V}$ to $1 \mathrm{~V}$, and for meshed polyester from $-10 \mathrm{~V}$ to $10 \mathrm{~V}$. The reason for the higher voltage used for the meshed polyester is that it is composed of many holes and pores, so that a higher voltage is required to obtain a flow of electrons.

The "conductivity" of silver-coated fabrics was calculated using Ohm's Law equation:

$$
I=\frac{V}{R}
$$

where $V$ is the potential difference measured across the resistance in units of volts; $I$ is the current through the fabric in units of amperes. and $R$ is the resistance of the conductor in units of ohms. Because the results of the "resistivity test", the appropriate voltage conditions for obtaining "high conductivity" of the two types of silver-coated polyester were found. For silver-coated mesh-polyester fabric, the "highest conductivity" value was measured, when one volt was applied to fabric, and for a piece of meshed-polyester fabric this value was found to be around $10 \mathrm{~V}$. These voltages were applied to the dye-loaded, PM-coated conductive fabrics to probe whether the dye can be released from the PMs attached to the surface of polyester.

The next stage in the preparation of the fabric is the sonochemical coating of "conductive fabrics" with dye-loaded proteinaceous microspheres. The creation and the anchoring of the dye-loaded microbubbles to the "conductive" fabrics were performed by a one-step sonochemical reaction. The effects of ultrasound on proteins was recently reviewed by Gedanken. ${ }^{8}$ After the sonochemical reaction there was a change in color of the solution, as shown in Figure2. The red color which is
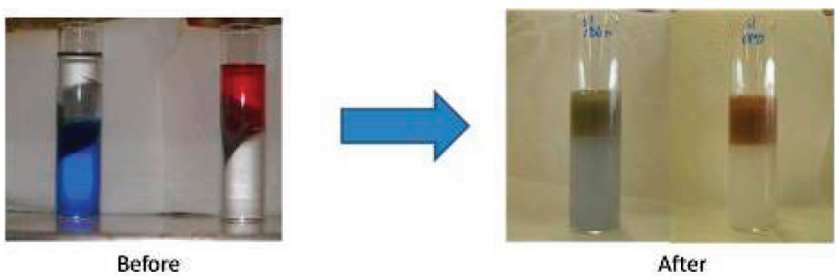

Figure 2. Sonochemical coating of "conductive fabrics" with dyeloaded proteinaceous microspheres (blue-RBBR; red-ORO). Left-side image $=$ solution before sonochemical reaction; right-side image $=$ solution after the sonochemical reaction.

emitted from the upper phase containing ORO (before sonochemical reaction) has almost disappeared, replaced by a slightly redish color emitted from the pahse containing the microspheres. The blue color (belongs to RBBR) became less intense after sonochemical reaction. The reason for this change is due to the encapsulation of the dye in the PMs.

As seen in Figure 2, the color of the microsphere layers (the upper layer) became slightly gray. This color change occurs because part of the silver coating (part of the silver nanoparticles attached to polyester) was released from the surface of the fabric and adhered to the other side of the microspheres'. The amount of dye encapsulated inside the PMs attached to the surface of coated polyester was calculated using values obtained from UV measurements and based on the Beer-Lambert law. The calculations were performed as follows. The amount of dye molecules (RBBR and ORO) loaded into the microspheres attached to the fabrics was calculated by subtracting, "free" dye molecules (molecules of dye which were not encapsulated inside the PMs, but found in the aqueous/ dodecane solution), and the dye molecules encapsulated in PMs which were not attached to the surface of polyester from the initial amount of dye introduced into the precursor mixture.

For the hydrophilic RBBR dye only $1.5 \%$ of the dye (from the initially introduced amount of dye) was found on the surface of silver-coated polyester. About $10 \%$ of the ORO dye was found inside the PMs attached to the surface of silvercoated polyester. The electrical charge and the size of resultant PMs were measured and compared with the electrical charge and size of the PMs produced when an uncoated piece of polyester was introduced into the precursor mixture of the reaction cell. The microspheres produced in the sonication cell with uncoated polyester have an electrical charge of $-32 \mathrm{meV}$ and an average size of about $117 \mathrm{~nm}$. The average size of the spheres formed in the reaction cell with silver-coated polyester is about $120 \mathrm{~nm}$ and the electrical charge is $+2 \mathrm{meV}$. There is no difference between the average sizes of the PMs produced in the presence of uncoated- or silver-coated polyester fabrics in the reaction cells. The main difference between the two types of 

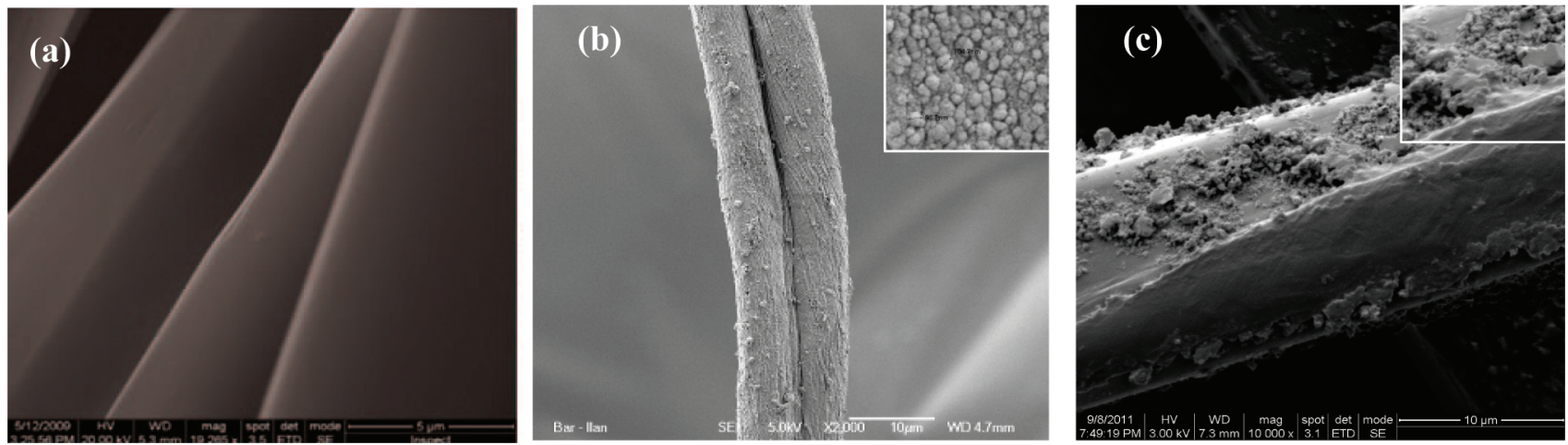

Figure 3. SEM micrographs of: (a) pristine polyester fibers, (b) fabrics coated with Ag nanoparticles. Reprinted with permission from ref 15 . Copyright 2008 Nanotechnology Publisher. (c) Polyester coated with silver nanoparticles and then coated with dye-loaded PMs. A higher magnification of each sample is presented on the upper right corner of each of the images. Scale bar $=10 \mu \mathrm{m}$.
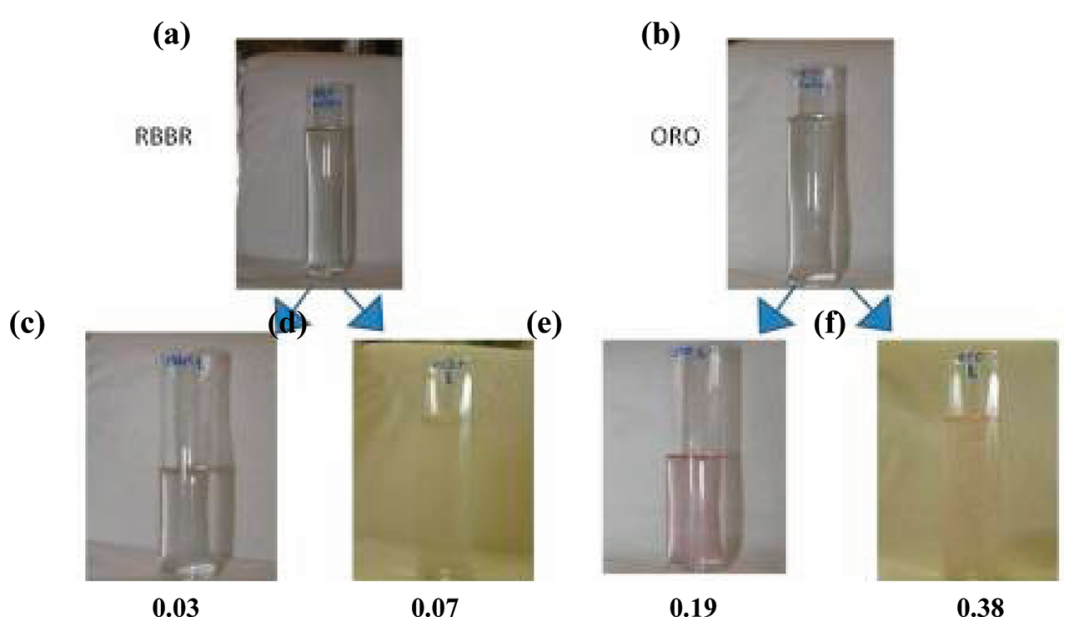

Figure 4. "Stability test" (stability of the attached microspheres in the reaction solution) presented in two upper images (no dye were found after 1.5 h). The results of the "release properties test"(stability of bonded spheres under electrical field) are presented in the four lower images (the ethanol solution was slightly colored with a pink color). Left, lowest curren; right, highest current.

PMs is their electric charge. In the case of PMs, which were formed in the presence of a coated fabric, the electric charge became positive, as compared with the negative charge of the bare spheres. This phenomenon could be explained by arguing that during sonochemical reaction (formation and attaching PMs) part of the silver nanoparticles were removed from the surface of the polyester and were attached to the outer surface of newly formed PMs.

The morphology of silver PM-coated polyester was studied using scanning electron microscopy analysis. The image in Figure $3 \mathrm{a}$ demonstrates the smooth structure of the fabric before coating with silver nanoparticles. Figure $3 b$ shows the image obtained from polyester yarn coated with silver nanoparticles. An enlarged image in the upper right corner of Figure $3 \mathrm{~b}$ is presented under higher magnification in order to observe the particles' size. The tendency of the particles is to form aggregates of $\sim 100 \mathrm{~nm}$ that consist of smaller nanoparticles of about $30 \mathrm{~nm}$. The aggregates were generated due to the high concentration of the nanoparticles that must achieve conductivity via the fabric. Figure $3 c$ depicts polyester coated with silver nanoparticles and then coated with dyeloaded PMs. The difference in the surface structure of 3 types of yarns was clearly observed. The PMs of BSA protein (see Figure 3c) covered the entire silver-coated surface of the polyester yarn (see enlarged image in the upper-right corner of
Figure 3c. Only a small portion of the silver nanoparticles was removed during sonochemical process, instead a large amount of the PMs were attached to the silver coated yarns. This explains the thick layer of the coating surface. The silver NPs layer is almost not seen under the thick layer of the newly formed and attached PMs.

The third stage of this project is the release of dye from the fabrics' surface by applying a potential across the fabric. For this purpose, PM-coated conductive fabrics were left for $1.5 \mathrm{~h}$ in the reaction solution. For the RBBR-loaded PMs, the "accepting" solution was $9 \%$ of a $\mathrm{NaCl}$ aqueous solution due to the dye being a hydrophilic agent. For ORO-loaded PM coatings, ethanol was used as a release examination solution. The aim of this experiment was to check if the destruction of dye-loaded PMs attached to fabrics was indeed caused by the electrical current and not by the testing solution, i.e., the dye diffuses through the walls of the PM to the testing solution. The result obtained was that no dye molecules leaked to the reaction solution in a $1.5 \mathrm{~h}$ examination. Figure $4 \mathrm{a}$ illustrates the "accepting" solution after soaking polyester coated with RBBR. On the right (Figure $4 \mathrm{~b}$ ) the ethanol solution is shown after soaking the polyester coated with ORO-loaded PMs for $1.5 \mathrm{~h}$. The testing solutions remain transparent, meaning that no dye was found in the "accepting" solutions after $1.5 \mathrm{~h}$ of soaking. The results of the same solutions after passing the current 
through the fabric are presented in Figures $4 c-f$. The values of UV absorption maxima at the wavelengths $640 \mathrm{~nm}$ (ORO) and $592 \mathrm{~nm}$ (RBBR) are presented under each image in Figure 4cf. The calculations of dye release (\%) were based on those values. The release test was performed by introducing electrodes on the surface of silver and PMs coated fabrics in the reaction solution and applying electrical current. Images $c$ and e in Figure 4 represent the solutions demonstrating their "release properties test" under a low voltage current of $0.1 \mathrm{~V}$. Applying a low voltage for $30 \mathrm{~min}$ through the ORO-containing fabric caused about $50 \%$ of the encapsulated ORO dye to be released from the spheres. When a higher voltage was applied $(10 \mathrm{~V})$ to the fabrics for $30 \mathrm{~min}$, the ethanol solution from ORO-coated polyester was colored with a distinct pink color (Figure 4f), detected by both the naked eye and by UV-vis measurements. This measurement shows that almost $100 \%$ of the encapsulated ORO dye was released from the polyester surface. On the other hand, no significant change in the "accepting" solution was detected for fabrics coated with RBBR-loaded PMs. The change in color after applying voltage to the coated fabrics with RBBR was not detected, neither by the naked eye nor with a UV-vis spectrophotometer.

The absence of RBBR from the examination solution after passing an electric current, could be explained by the fact that the concentration of the hydrophilic dye in the PMs is much lower than the concentration of the hydrophobic dye, so that when the current flows through the fabric it decomposes the spheres in both cases. However, when PMs are filled with the hydrophobic ORO, there is almost no RBBR in the spheres as a result of its hydrophilic nature. The hydrophobic dye (ORO) due to sonochemical encapsulation goes inside the dodecane filled BSA spheres and hydrophilic dye (RBBR) adsorbed to the hydrophilic part of the spheres, which are outer surface of BSA PMs. This behavior explains the results obtained for yield of encapsulation and yield of release by electrical current of ORO and RBBR dyes.

\section{CONCLUSION}

In the current research, we applied ultrasonic waves to produce silver-coated "conductive" fabrics and to coat those fabrics with dye-loaded PMs. The conductivity of the fabrics was used to control the dye release of the coated fabrics. Two types of dyes were used to study the release properties of conductive polyester fabrics To summarize the results, the hydrophobic dye (ORO) was successfully encapsulated within the PMs, which were attached to the surface of a "conductive" polyester fabric. The ORO was released from the fabric's surface by applying an electric current through the fabric. The fabrics were examined after $30 \mathrm{~min}$ under the voltage of $1 \mathrm{~V} / 10 \mathrm{~V}$, and it was found that for both dyes most of the microspheres were destroyed. Only very few PMs survived the current. In the future, these PM-coated conductive fabrics could be used in medicine for drug release. The encapsulated dye can be replaced with a drug that could be released from the surface of fabrics by applying a low voltage.

Due to the hydrophobic nature of the polyester yarn and the hydrophobicity of the inner part of the attached PMs, the most suitable dye for encapsulation and release was the hydrophobic dye, ORO.

\section{AUTHOR INFORMATION}

\section{Corresponding Author}

*E-mail: gedanken@mail.biu.ac.il.

\section{Notes}

The authors declare no competing financial interest.

\section{REFERENCES}

(1) Gedanken., A. Ultrason. Sonochem. 2004, 11, 47-55.

(2) Kotlyar, A.; Perkas, N.; Amiryan, G.; Meyer, M.; Zimmermann, W.; Gedanken., A. J. Appl. Polym. Sci. 2007, 104, 2868-2876.

(3) Pol, V. G.; Srivastava, D. N.; Palchik, O.; Palchik, V.; Slifkin, M. A.; Weiss, A.; M.; Gedanken, A. Langmuir 2002, 18, 3352-3357.

(4) Pol, V. G.; Wildermuth, G.; Felsche, J.; Gedanken, A.; CalderonMoreno., J. J. Nanosci. Nanotechnol. 2005, 5, 975-979.

(5) Perkas, N.; Amirian, G.; Dubinsky, S.; Gazit, S.; Gedanken., A. J. Appl. Polym. Sci. 2007, 104, 1423-1430.

(6) Angel (Shimanovich), U.; Silva, C. M.; Cavaco-Paulo, A.; Gedanken., A. Isr. J. Chem. 2010, 50, 524-529.

(7) Shimanovich, U.; Cavaco-Paulo, A.; Nitzan, Y.; Gedanken., A. Chem.-Eur. J. 2012, 18, 365-369.

(8) Gedanken., A. Chem.-Eur. J. 2008, 14, 3840-3853.

(9) Shchukin, D. G.; Möhwald, H. Phys. Chem. Chem. Phys. 2006, 8, 3496-3506.

(10) Grinstaff, M. W.; Suslick, K. S. Proc. Natl. Acad. Sci. U.S.A. 1991, $88,7708-7710$

(11) Suslick, K. S.; Grinstaff, M. W.; Kolbeck, K. A.; Wong., M. Ultrason. Sonochem. 1994, 1, S65-S68.

(12) Trivedi, K. N.; Boricha, A. B.; Bajaj, H. C.; Jasra., R. V. Rasayan J. Chem. 2009, 2, 379-385.

(13) Ramirez-Zacarias, J. J. L.; Castro-Mufiozledo, F.; Kuri- Harcuch., W. Histochemistry 1992, 97, 493-497.

(14) Vogel., A. I. A Textbook of Quantitative Inorganic Analysis: Theory and Practice; Longman: London, 1962; p 256.

(15) Perelshtein, I.; Applerot, G.; Perkas, N.; Guibert, G.; Mikhailov, S.; Gedanken, G. Nanotechnology 2008, 19, 245705-245711. 